\title{
Correction to: Advancing Healthcare Through Personalized Medicine
}

\section{Correction to: \\ P. Hays, Advancing Healthcare Through Personalized Medicine, https://doi.org/10.1007/978-3-030-80100-7}

The company name that had appeared in the copyright page as "Personalis, Inc" has been changed to "Hays Documentation Specialists, LLC". 